\title{
Roughness-induced critical phenomena in a turbulent flow
}

\author{
Nigel Goldenfeld \\ Department of Physics, University of Illinois at Urbana-Champaign, \\ 1110 West Green Street, Urbana, Illinois, 61801-3080.
}

\begin{abstract}
I present empirical evidence that turbulent flows are closely analogous to critical phenomena, from a reanalysis of friction factor measurements in rough pipes. The data collapse found here corresponds to Widom scaling near critical points, and implies that a full understanding of turbulence requires explicit accounting for boundary roughness.

PACS numbers: 47.27.Jv, 47.27.Nz, 89.75.Da, 05.70.Jk
\end{abstract}

Turbulent phenomena in fluids 1] are characterized by strong fluctuations and power-law spectra 2 that are suggestive [3] of the power-law correlations observed in critical phenomena at continuous phase transitions [4]. Attempts to link these two sets of phenomena have primarily, but not exclusively [5, 6], focused on the calculation of scaling exponents [7] in ideal systems which are isotropic and homogeneous, neglecting boundaries. However, no analogue has been found for the wide variety of thermodynamic scaling phenomena [ [] that are an equally fundamental aspect of continuous phase transitions, and whose elucidation 9 led to a complete understanding of critical phenomena 10]. Here we consider the important role of boundary roughness on fluid flow, by reanalyzing Nikuradse's experimental measurements 11] of the friction exerted on a turbulent fluid by the walls of a rough pipe. We show that the data as a function of Reynolds number and relative roughness collapse onto one universal curve, when appropriately scaled. This analogue of Widom scaling [8] implies that boundary roughness must be included in a complete description of turbulence, and establishes the long sought-after precise connection to critical phenomena.

Turbulent flows are characterised by their Reynolds number, defined as $R e \equiv U L / \nu$, where $U$ is a typical velocity at the length scale $L$, and $\nu$, the kinematic viscosity, is the viscosity of the fluid divided by its density. In 1941, Kolmogorov 12 and Obukhov 13], recognised that at large enough Reynolds numbers, fluid motion is, over a wide range of length scales, a dynamical, energy-conserving but irregular swirling motion [1] governed by inertia, rather than a dissipative phenomenon. Thus, they pointed out that for this so-called inertial range of scales, observables should be independent of $\nu$. In particular, for the inertial range, the turbulent energy spectrum $E$ of longitudinal velocity fluctuations $\delta v_{k}$ in wavenumber space, $k$, can only depend upon the mean energy transfer rate $\bar{\epsilon}$ and $k$ itself in a manner dictated by dimensional analysis: $E(k) \equiv\left\langle\left|\delta v_{k}\right|^{2}\right\rangle=\bar{\epsilon} k^{-5 / 3}$. This experimentally-verified 14 power-law scaling (often referred to as K41) applies on small scales in a turbulent flow, but not so small that molecular viscosity becomes important. The existence of a wide range of length scales, over which power-law (and thus scale-invariant) correlated fluctuations are found, is reminiscent 3 of the power-law fluctuations on many length scales that accompany critical phenomena [4]: for example, in a ferromagnet near its critical point, the Fourier component of the magnetisation $M$ at wavenumber $k$ satisfies $G(k) \equiv\left\langle\left|M_{k}\right|^{2}\right\rangle \propto k^{-2+\eta}$, where $\eta$ is an anomalous scaling exponent that describes departures from mean field theory. This power-law scaling applies at large scales, and is independent of the small scale details of the system, such as the nature of the crystal lattice.

Power-law scaling of correlation functions is, however, only one of two key aspects of critical phenomena [4]. The other, equally important aspect is the phenomenon of data collapse, or Widom scaling [8]: for example, in a ferromagnet, the equation of state, nominally a function of two variables, is expressible in terms of a single reduced variable that depends on a combination of external field and temperature. What is the turbulent analogue to Widom scaling in thermodynamics? To address this question, it is necessary to examine data on the largescale properties of turbulence, for example the friction factor in pipe flow.

In 1932 and 1933, Nikuradse undertook a seminal series of measurements of flow in nominally smooth 15 and rough pipes 11, measuring inter alia the friction factor $f$, related to the pressure drop across the pipe 16, 17]. These measurements have remained the benchmark in the field, being the only systematic measurements of a single flow geometry over such a wide range of Reynolds numbers. Nikuradse's experiments on sand-roughened pipes used sand grains of a well-defined size $r$, repeated over a wide range of values of $r$ and pipes of different radii $D$. Nikuradse was able to verify the expectation of hydrodynamic similarity: the flow properties depend on the roughness only through the combination $r / D$. Nikuradse presented results for the shear force per unit area $\tau$ exerted by the flow on the walls of the pipe in the form $f=\tau / \rho U^{2}$, and these data are plotted in figure (11).

There are several broad features to note in these data. First, in the smoothest pipes studied, for $R e<100,000$, in the turbulent regime, the friction factor is a decreasing function of $R e$, varying to a good approximation 


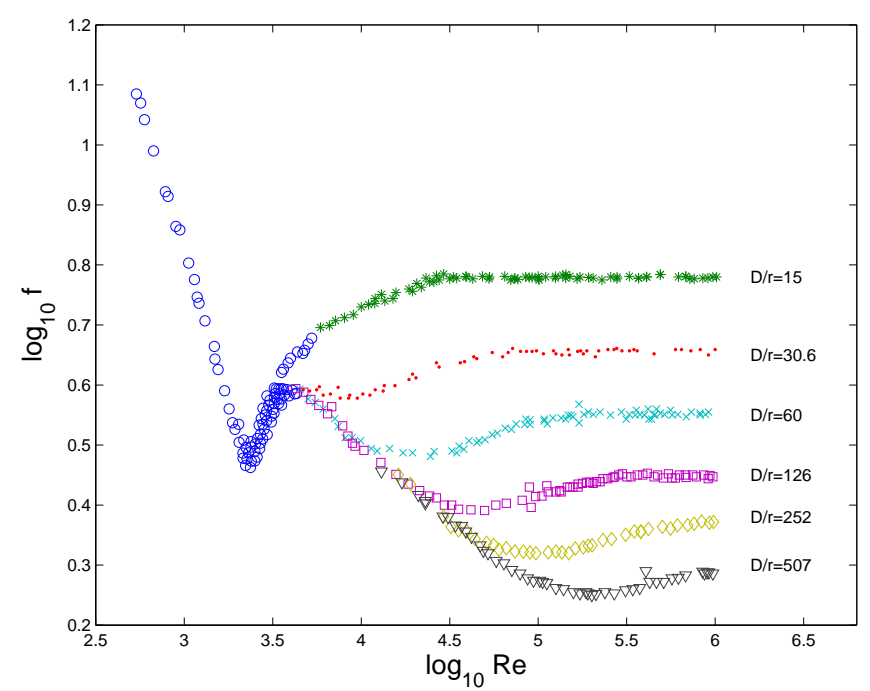

FIG. 1: (Color online) Friction factor for turbulent flow in a rough pipe, as reported by Nikuradse 11]. The data were measured at different values of $R e$ and $r / D$, and extracted from Nikuradse's tabular and graphical presentation.

in a manner usually attributed to Blasius 18, 19] as $f \sim R e^{-1 / 4}$. As the roughness scale is increased, and thus for smaller values of $D / r$, the extent over which the Blasius scaling extends becomes smaller. Thus, we can represent this feature by the statement that $f \sim R e^{-1 / 4}$ asymptotically as $r / D \rightarrow 0$. Asymptotically for rough pipes at large $R e$, the friction becomes independent of $R e$, and depends only on the roughness $r / D$, varying to a good approximation, according to Strickler's law [20], as $f \sim(r / D)^{1 / 3}$. These broad characteristics are also visible in aggregate in other pipe flow data 21, 22, 23, 24, 25], but no other single data set captures the full range shown here, with as little scatter evident in the data.

These features place strong constraints on the functional form of the friction factor $f(R e, r / D)$. In fact, these constraints precisely parallel those on thermodynamic properties of ferromagnets near the critical temperature $T_{c}$, as a function of reduced temperature $t=$ $\left|T-T_{c}\right| / T_{c}$ and external magnetic field $H$. For example, at the critical temperature, the magnetic equation of state has the form $M \sim H^{1 / \delta}$ for $t=0$, where $\delta$ is a critical exponent whose value can be computed by renormalisation group (RG) theory [4]; and for zero field, the magnetisation continuously approaches zero as $T \rightarrow T_{c}{ }^{-}$ with a power law variation $M \sim t^{\beta}$ for $H=0$, where $\beta$ is another critical exponent whose value can be computed by RG. Widom [8] discovered that these properties all followed if the thermodynamic free energy obeyed certain scaling properties, later shown to follow from renormalization group considerations 9,10 .

We connect scaling in turbulence with that in critical phenomena by observing that the limit $R e \rightarrow \infty$ is analogous to the limit $t^{-1} \rightarrow \infty$, whereas the limit $H \rightarrow 0$

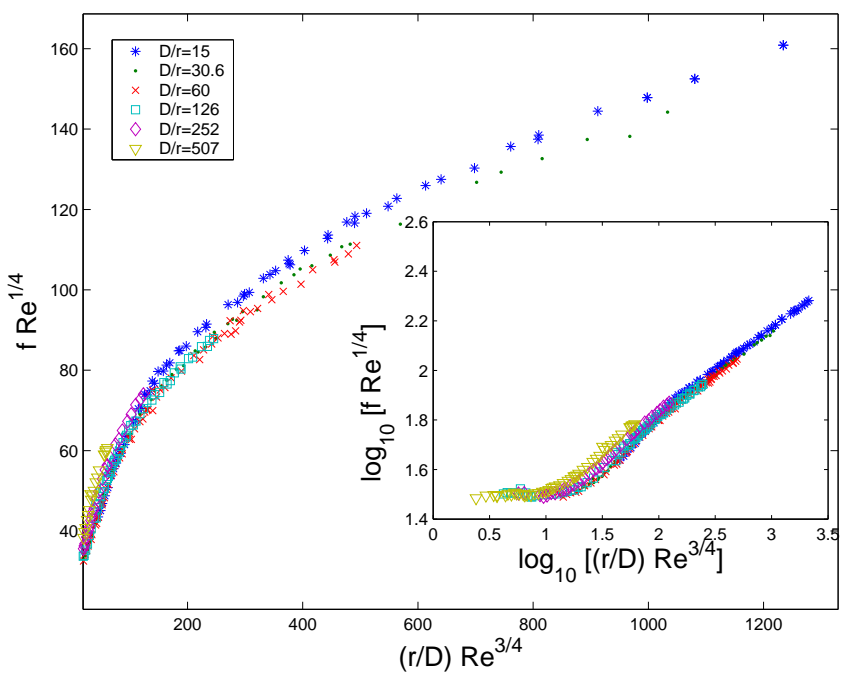

FIG. 2: (Color online) Friction factor for turbulent flow in a rough pipe, as reported by Nikuradse 11], scaled according to the text. Inset: the same plot on a logarithmic scale.

is analogous to the limit $r / D \rightarrow 0$. Thus, the analytic properties of the friction factor can be derived if we follow Widom's scaling argument [8], and propose a scaling form for the friction factor: $f(R e, r / D)=R e^{-1 / 4} g\left(R e^{\alpha} r / D\right)$, where $g(z)$ is an unknown scaling function of a single variable $z$, which tends to a constant for small values of $z$, and $\alpha$ is an exponent that we can determine by the requirement that the $R e$ dependence should cancel out of the formula for $f$ at large $R e$, leaving the Strickler law $f \sim(r / D)^{1 / 3}$. This requires that $g(z) \sim z^{1 / 3}$ as $z \rightarrow \infty$, and therefore $\alpha / 3=1 / 4$. Thus, we conclude that

$$
f=R e^{-1 / 4} g\left(R e^{3 / 4}(r / D)\right) .
$$

The scaling form of Eq. (11) predicts that the turbulent friction factor data, measured as a function of both $r / D$ and $R e$, and thus in principle occupying a two dimensional space, will actually collapse onto a one-dimensional curve, when plotted as $f R e^{1 / 4}$ versus $(r / D) R e^{3 / 4}$. The test of this prediction is shown in figure (2), where Nikuradse's data occupying the plane of figure (1) collapses onto a single curve when plotted in the reduced variables of Eq. (11). Note that the data collapse occurs for those data that lie between the Blasius and Strickler regimes only. Small deviations from the data collapse are visible, but it is not clear to what extent these reflect uncertainties in the data 26] or something more fundamental.

The scaling function that we have extracted from the data is unlikely to be universal in the sense of being independent of the nature of the roughness of the pipe. By analogy with the effects of long-range interactions in critical spin systems [4], it seems probable that self-affine roughness 25 will have a different effect on the flow than periodic single-scale roughness, and this can be reflected in the scaling function, the scaling exponents, or both. 
Inspection of the data from different pipe flow experiments suggests that the first possibility is the most likely, but this remains to be checked in detail.

The interpretation of the analogy discovered here is very natural. In magnetic systems, for example, the power-law scaling of $G(k)$ is now understood to be associated with the fact that magnetisation can be induced in a magnet by the application of an external magnetic field $H$. The sensitivity of the order in a magnet to a perturbation by $H$ becomes exquisite near a critical point, and thermodynamic variables contain a singular dependence on $H$ and $t$. The fact that the boundary roughness plays the role of external magnetic field is a reflection of the fact that small perturbations couple to the flow and drive the turbulent state.

The exponents in the scaling theory given here are taken from experiment; however, Gioia and Chakraborty have recently pointed out 27 that the friction factor $f$ can be related to the local structure of turbulence, by considering the momentum flux at the pipe boundary. The Nikuradse data show four features: a hump, the Blasius regime, a shallow minimum, and the Strickler regime. The scaling argument presented here implies that the Blasius and Strickler regimes are both manifestations of inertial range scaling coupled with wall friction, and indeed, Gioia and Chakraborty find from momentum flux considerations that this is sufficient to reproduce the Blasius and Strickler regimes. If they then include the dissipation range in their formula for the friction factor, they find that this reproduces the shallow minimum between the Blasius and Strickler regime. Interesting their formulae for the pure inertial and the inertial + dissipation ranges both satisfy Eq. (11). The hump in the friction factor arises from the energy-containing range, lies outside the range bracketed by Blasius and Strickler and is absent from their predictions if this spectral feature is not included in their formula. Thus, in summary, the features which are described by Eq. (II) are indeed related to the spectral features of the inertial range (and the dissipation range). Thus, just as in critical phenomena, the large-scale phenomenology of turbulence can be related to the power law fluctuations. Presumably, the scaling result Eq. (11) can be derived from a renormalization group argument 9 .

Eq. (11) describes the nonequilibrium driven steady state of fully-developed turbulence, which is to be contrasted with the phenomenon of the transition from laminar to turbulent flow. In the case of turbulent pipe flow, it is generally accepted that laminar HagenPoiseuille flow is linearly stable at all Reynolds numbers, and that there is a finite amplitude instability to turbulence 28, 29], whose amplitude varies [30] as $R e^{-1}$. Thus, this transition has the character of a first order transition in thermodynamics, and is not related to the theory given here.

Our results establish boundary roughness as a key el- ement in a proper theoretical description of turbulence, in the same way that a proper understanding of the ferromagnetic critical point at zero field would not be possible without taking into account the behaviour for nonzero values of $H$. Our analysis highlights the need for a definitive set of experiments to replicate Nikuradse's data set, with a view to greater precision and removing sources of uncertainty 26] in both the data set and in the widely-used Colebrook 24] semi-empirical fit 31]. In ongoing work we are exploring the effects of roughness in two-dimensional turbulence 32], where predictions for the analogues of the Blasius and Strickler laws reveal interesting differences from the three dimensional results.

I acknowledge valuable discussions with G.I. Barenblatt, Gustavo Gioia and Pinaki Chakraborty, whom I also thank for assistance with the figures. This work was supported in part by the National Science Foundation through grant number NSF-EAR-02-21743.

[1] K. R. Sreenivasan, Rev. Mod. Phys. 71, S383 (1999).

[2] U. Frisch, Turbulence: The Legacy of A.N. Kolmogorov (Cambridge University Press, 1995).

[3] G. Eyink and N. Goldenfeld, Phys. Rev. E 50, 4679 (1994).

[4] N. Goldenfeld, Lectures on phase transitions and the renormalization group (Addison-Wesley, 1992).

[5] A. J. Chorin, Vorticity and turbulence (Springer, 1997).

[6] S. T. Bramwell, P. C. W. Holdsworth, and J. F. Pinton, Nature 396, 552 (1998).

[7] K. R. Sreenivasan and R. A. Antonia, Annu. Rev. Fluid Mech. 29, 435 (1997).

[8] B. Widom, J. Chem. Phys. 43, 3898 (1965).

[9] L. P. Kadanoff, Physics 2, 263 (1966).

[10] K. G. Wilson, Phys. Rev. B 4, 3174 (1971).

[11] J. Nikuradze (1933), vDI Forschungsheft, vol. 361 [In English, in Technical Memorandum 1292, National Advisory Committee for Aeronautics (1950).].

[12] A. N. Kolmogorov, Dokl. Akad. Nauk. SSSR 30, 299 (1941), [English translation in Proc. R. Soc. London Ser. A 434 (1991)].

[13] A. M. Obukhov, Dokl. Akad. Nauk. SSSR 32, 22 (1941).

[14] H. L. Grant, R. W. Stewart, and A. Moilliet, J. Fluid Mech. 12, 241 (1962).

[15] J. Nikuradze (1932), vDI Forschungsheft, vol. 356 [In English, in NASA TT F-10, 359 (1966).].

[16] J. Jiménez, Ann. Rev. Fluid Mech. 36, 173 (2004).

[17] B. J. McKeon, M. V. Zagarola, and A. J. Smits, J. Fluid Mech. 538, 429 (2005).

[18] H. Blasius (1913), forsch. Arb. Ing. Wes. No. 134, Berlin.

[19] H. Schlichting, Boundary layer theory (McGraw-Hill, 1979).

[20] A. Strickler (1923), mitteilungen des Eidgenossischen Amtes fur Wasserwirtschaft 16, Bern, Switzerland Translated as "Contributions to the question of a velocity formula and roughness data for streams, channels and closed pipelines." by T. Roesgan and W. R. Brownie, Translation T-10, W. M. Keck Lab of Hydraulics and Water Resources, Calif. Inst. Tech., Pasadena, Calif. Jan- 
uary 1981.

[21] B. Bauer and F. Galavics, Archiv für Waermewirtschaft 17, 125 (1936).

[22] F. Galavics, Schweizer Archiv 5, 337 (1939).

[23] C. F. Colebrook and C. M. White, Proc. Roy. Soc. Lon, Ser. A 161, 367 (1937).

[24] C. F. Colebrook, Institution of Civ. Eng. Journal 11, 133 (1939).

[25] E. Sletfjerding and J. S. Gudmundsson, J. Energy Res. Tech. 125, 126 (2003).

[26] J. Williamson, La Houille Blanche 6, 738 (1951).

[27] G. Gioia and P. Chakraborty (2005), submitted to Physical Review Letters. Available at http://arxiv.org/abs/physics/0507066.

[28] W. Pfenniger, in Boundary Layer and Flow Control, edited by G. V. Lachman (Pergamon, New York, 1961), pp. 961-980.

[29] B. Hof et al., Science 305, 1594 (2004).

[30] B. Hof, A. Juel, and T. Mullin, Phys. Rev. Lett. 91, 2445021 (2003).

[31] A. J. Smits, M. Shockling, and J. Allen, in 4th AIAA Theoretical Fluid Mechanics Meeting (2005).

[32] H. Kellay and W. I. Goldburg, Rep. Prog. Phys. 65, 845 (2002). 